\title{
Interaction Between the Broad-lined Type Ic Supernova 2012ap and Carriers of Diffuse Interstellar Bands
}

\section{Citation}

Milisavljevic, Dan, Raffaella Margutti, Kyle N. Crabtree, Jonathan B. Foster, Alicia M. Soderberg, Robert A. Fesen, Jerod T. Parrent, et al. 2014. "INTERACTION BETWEEN THE BROAD-LINED TYPE Ic SUPERNOVA 2012ap AND CARRIERS OF DIFFUSE INTERSTELLAR BANDS." The Astrophysical Journal 782 (1): L5. https://doi.org/10.1088/2041-8205/782/1//5.

\section{Permanent link}

http://nrs.harvard.edu/urn-3:HUL.InstRepos:41412098

\section{Terms of Use}

This article was downloaded from Harvard University's DASH repository, and is made available under the terms and conditions applicable to Other Posted Material, as set forth at http:// nrs.harvard.edu/urn-3:HUL.InstRepos:dash.current.terms-of-use\#LAA

\section{Share Your Story}

The Harvard community has made this article openly available.

Please share how this access benefits you. Submit a story.

\section{Accessibility}




\title{
INTERACTION BETWEEN THE BROAD-LINED TYPE Ic SUPERNOVA 2012ap AND CARRIERS OF DIFFUSE INTERSTELLAR BANDS
}

\author{
Dan Milisavljevic ${ }^{1}$, Raffaella Margutti ${ }^{1}$, Kyle N. Crabtree $^{1}$, Jonathan B. Foster ${ }^{2}$, Alicia M. Soderberg ${ }^{1}$, \\ Robert A. Fesen ${ }^{3}$, Jerod T. Parrent ${ }^{3,4}$, Nathan E. Sanders $^{1}$, Maria R. Drout ${ }^{1}$, Atish Kamble ${ }^{1}$, Sayan Chakraborti $^{1}$, \\ Timothy E. Pickering ${ }^{5,6}$, S. Bradley Cenko ${ }^{7,8}$, Jeffrey M. Silverman ${ }^{9}$, Alexei V. Filippenko ${ }^{8}$, Robert P. Kirshner ${ }^{1}$, \\ Paolo Mazzali ${ }^{10,11,12}$, KeiIChi Maeda ${ }^{13,14}$, G. H. Howie Marion ${ }^{9}$, Jozsef Vinko ${ }^{9,15}$, and J. Craig Wheeler ${ }^{9}$ \\ ${ }^{1}$ Harvard-Smithsonian Center for Astrophysics, 60 Garden St., Cambridge, MA 02138, USA; dmilisav@ cfa.harvard.edu \\ ${ }^{2}$ Yale Center for Astronomy and Astrophysics, Yale University, New Haven, CT 06520, USA \\ ${ }^{3}$ Department of Physics \& Astronomy, Dartmouth College, 6127 Wilder Lab, Hanover, NH 03755, USA \\ ${ }^{4}$ Las Cumbres Observatory Global Telescope Network, Goleta, CA, USA \\ 5 Southern African Large Telescope, P.O. Box 9, Observatory 7935, Cape Town, South Africa \\ ${ }^{6}$ Space Telescope Science Institute, 3700 San Martin Drive, Baltimore, MD 21218, USA \\ ${ }^{7}$ Astrophysics Science Division, NASA Goddard Space Flight Center, Mail Code 661, Greenbelt, MD 20771, USA \\ 8 Department of Astronomy, University of California, Berkeley, CA 94720-3411, USA \\ ${ }^{9}$ University of Texas at Austin, 1 University Station C1400, Austin, TX 78712-0259, USA \\ ${ }^{10}$ Astrophysics Research Institute, Liverpool John Moores University, Liverpool L3 5RF, UK \\ ${ }^{11}$ Max-Planck-Institut für Astrophysik, Karl-Schwarzschild-Strasse 1, D-85748 Garching, Germany \\ 12 INAF-Osservatorio Astronomico di Padova, Vicolo dell'Osservatorio 5, I-35122, Padova, Italy \\ ${ }^{13}$ Department of Astronomy, Kyoto University Kitashirakawa-Oiwake-cho, Sakyo-ku, Kyoto 606-8502, Japan \\ ${ }^{14}$ Kavli Institute for the Physics and Mathematics of the Universe (WPI), Todai Institutes for Advanced Study, University of Tokyo, \\ 5-1-5 Kashiwanoha, Kashiwa, Chiba 277-8583, Japan \\ 15 Department of Optics and Quantum Electronics, University of Szeged, Domter 9, 6720, Szeged, Hungary \\ Received 2013 October 17; accepted 2014 January 3; published 2014 January 21
}

\begin{abstract}
Diffuse interstellar bands (DIBs) are absorption features observed in optical and near-infrared spectra that are thought to be associated with carbon-rich polyatomic molecules in interstellar gas. However, because the central wavelengths of these bands do not correspond to electronic transitions of any known atomic or molecular species, their nature has remained uncertain since their discovery almost a century ago. Here we report on unusually strong DIBs in optical spectra of the broad-lined Type Ic supernova SN 2012ap that exhibit changes in equivalent width over short ( $\lesssim 30$ days) timescales. The $4428 \AA$ and $6283 \AA$ DIB features get weaker with time, whereas the $5780 \AA$ feature shows a marginal increase. These nonuniform changes suggest that the supernova is interacting with a nearby source of DIBs and that the DIB carriers possess high ionization potentials, such as small cations or charged fullerenes. We conclude that moderate-resolution spectra of supernovae with DIB absorptions obtained within weeks of outburst could reveal unique information about the mass-loss environment of their progenitor systems and provide new constraints on the properties of DIB carriers.
\end{abstract}

Key words: astrochemistry - ISM: lines and bands - ISM: molecules - molecular processes - supernovae: general - supernovae: individual (SN 2012ap)

Online-only material: color figures

\section{INTRODUCTION}

One of the oldest unsolved problems in optical and infrared astronomy is the nature of diffuse interstellar bands (DIBs). DIBs represent more than 400 absorption features observed in optical and near-infrared spectra that are typically narrow (FWHM intensity $<1 \AA$ ) and weak (less than $5 \%$ below the continuum), with central wavelengths that do not correspond to any known atomic or molecular species (Herbig 1995; Hobbs et al. 2009; Geballe et al. 2011). They were first noticed in stellar spectra by Heger (1922). Merrill (1934) subsequently uncovered a number of DIBs as ubiquitous interstellar features and their nature has been an enduring subject of speculation.

It is now well established that sources (or "carriers") of DIBs are found in the interstellar medium (ISM). DIB features remain stationary in spectroscopic binaries, and there are rough correlations between extinction and $\mathrm{Na}$ I $\mathrm{D}$ column density with the intensity of DIB features (Herbig 1995). Searches for DIBs in circumstellar shells have generally reported null detections or results that cannot distinguish whether the absorption arises in circumstellar material or the intervening ISM (Snow \& Wallerstein 1972; Luna et al. 2008).

Merrill (1934) was the first to suspect dust grains and/ or molecules as possible carriers of DIBs. After nearly a century of observational, theoretical, and experimental work, these two original suggestions have remained the primary candidates, occasionally swapping in popularity (see Sarre 2006 and references therein). Current research favors multiple carriers produced by a mix of fairly large and complex carbon-based ("organic") polyatomic molecules composed of cosmically abundant elements such as $\mathrm{H}, \mathrm{C}, \mathrm{O}$, and $\mathrm{N}$. There has been considerable investigation of polycyclic aromatic hydrocarbons (PAHs) as DIB carriers, but as yet no firm associations between PAH species and DIB features have been found (see Cox 2011 for a recent review).

Insights into the chemical and physical properties of DIB carriers have come from the study of their behavior in different interstellar environments, especially extragalactic ones. Most studies have focused on nearby star systems including the Magellanic Clouds and M31 (Cox et al. 2007; Cordiner et al. 


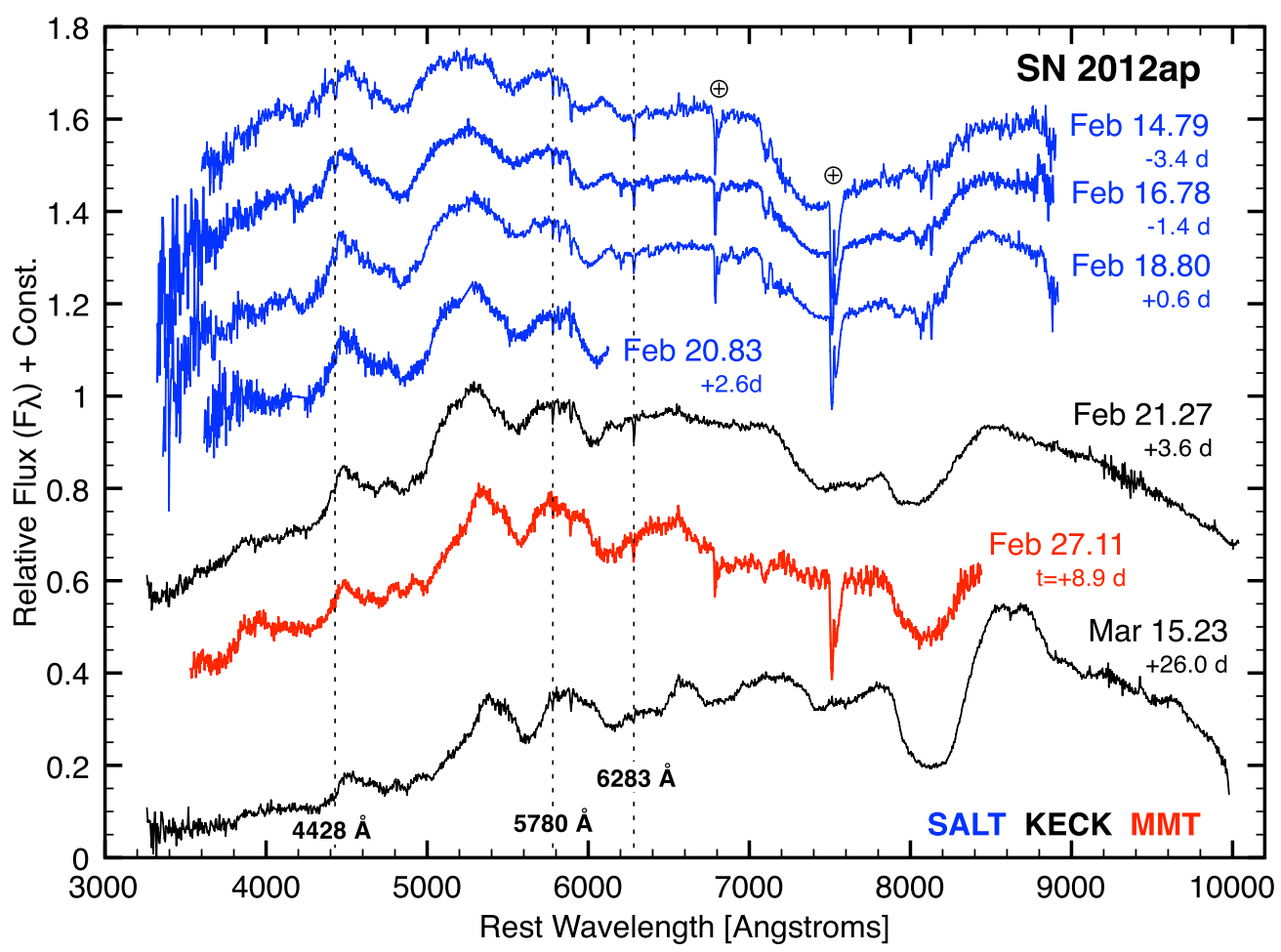

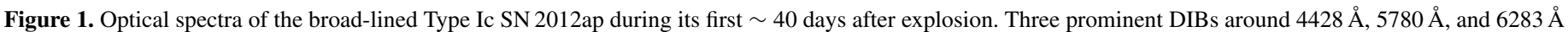

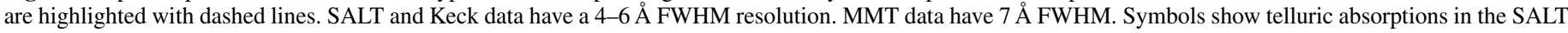

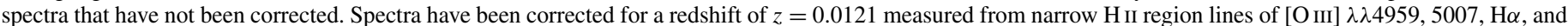
[N II] $\lambda \lambda 6548,6583$ observed near the location of the supernova.

(A color version of this figure is available in the online journal.)

2008). Outside of limited work with quasars (e.g., Ellison et al. 2008), only supernovae (SNe) have been bright enough to probe DIBs beyond the Local Group (see, e.g., Cox \& Patat 2008). In general, extragalactic studies have shown that DIB carrier abundances can be similar to Galactic values, though systematic differences sometimes exist.

In this Letter we report on recent observations of a broadlined Type Ic SN that exhibits some of the strongest DIBs ever detected in an extragalactic source. These absorptions undergo changes in intensity over relatively short timescales in a manner that suggests that the $\mathrm{SN}$ explosion interacted with local carriers of DIBs. We conclude that moderate-resolution spectra of the SNe obtained shortly after outburst may provide a new and powerful probe of DIBs and offer clues about the progenitor systems of these explosions.

\section{RESULTS}

\subsection{Discovery and Classification}

SN 2012ap was first detected by the Lick Observatory Supernova Search at coordinates $\alpha(2000.0)=05^{\mathrm{h}} 00^{\mathrm{m}} 13.72$ and $\delta(2000.0)=-03^{\circ} 20^{\prime} 51^{\prime \prime} .2$ in the face-on galaxy NGC 1729 $(d \approx 43.1 \mathrm{Mpc}$; Springob et al. 2009) on February $10.23 \mathrm{UT}$ (Jewett et al. 2012). The SN is located in the outskirts of the host galaxy some $7.1 \mathrm{kpc}$ in projection from the nucleus in a region with no obvious star formation.

The first reports of optical spectra of SN 2012ap classified it as a Type Ib/c SN similar to SN 2008D not long after explosion (Xu et al. 2012). This prompted extensive follow-up observations by our group that included optical spectra obtained with the $10 \mathrm{~m}$ Southern African Large Telescope (SALT) using the Robert Stobie Spectrograph (Burgh et al. 2003), the 10 m Keck-I telescope using the Low Resolution Imaging Spectrometer (Oke et al. 1995), and the MMT $6.5 \mathrm{~m}$ telescope using the Blue Channel spectrograph (Schmidt et al. 1989). The spectra shown in Figure 1 are part of a larger data set (D. Milisavljevic et al., in preparation).

Unlike SN 2008D, which transitioned to a Type Ib SN exhibiting conspicuous He I, the spectra of SN 2012ap obtained weeks later continued to show broad features associated with ejecta traveling $\sim 2 \times 10^{4} \mathrm{~km} \mathrm{~s}^{-1}$. Milisavljevic et al. (2012) reported that these later spectra were similar to those observed in broad-lined SN Ic such as SN 1998bw and SN 2002ap 1-2 weeks after maximum light (see Figure 1). Further examination shows that the later spectra of SN 2012ap also resemble those of $\mathrm{SN} 2009 \mathrm{bb}$, an SN Ib/c that had a substantial relativistic outflow powered by a central engine (Soderberg et al. 2010; Pignata et al. 2011).

\subsection{Strong DIB Features}

Superimposed on the broad-lined Type Ic features of SN 2012ap are conspicuous absorptions with equivalent widths (EWs) $\gtrsim 1 \AA$ associated with DIBs at the rest wavelength of the host galaxy. The DIB features are strongest at $4428 \AA, 5780 \AA$, and $6283 \AA$, which are the wavelengths of well-known DIBs typically seen in stellar spectra (Herbig 1995). In Figure 2 we display enlarged regions around these features. Not shown is another possible DIB detection near $6203 \AA$ that may be contaminated by an $\mathrm{OH}$ telluric line at an observed wavelength of $6280 \AA$ A.

The central wavelengths of these DIBs do not change with time, but the intensities do exhibit measurable changes that are not uniform across different features (Figure 2, right column). 

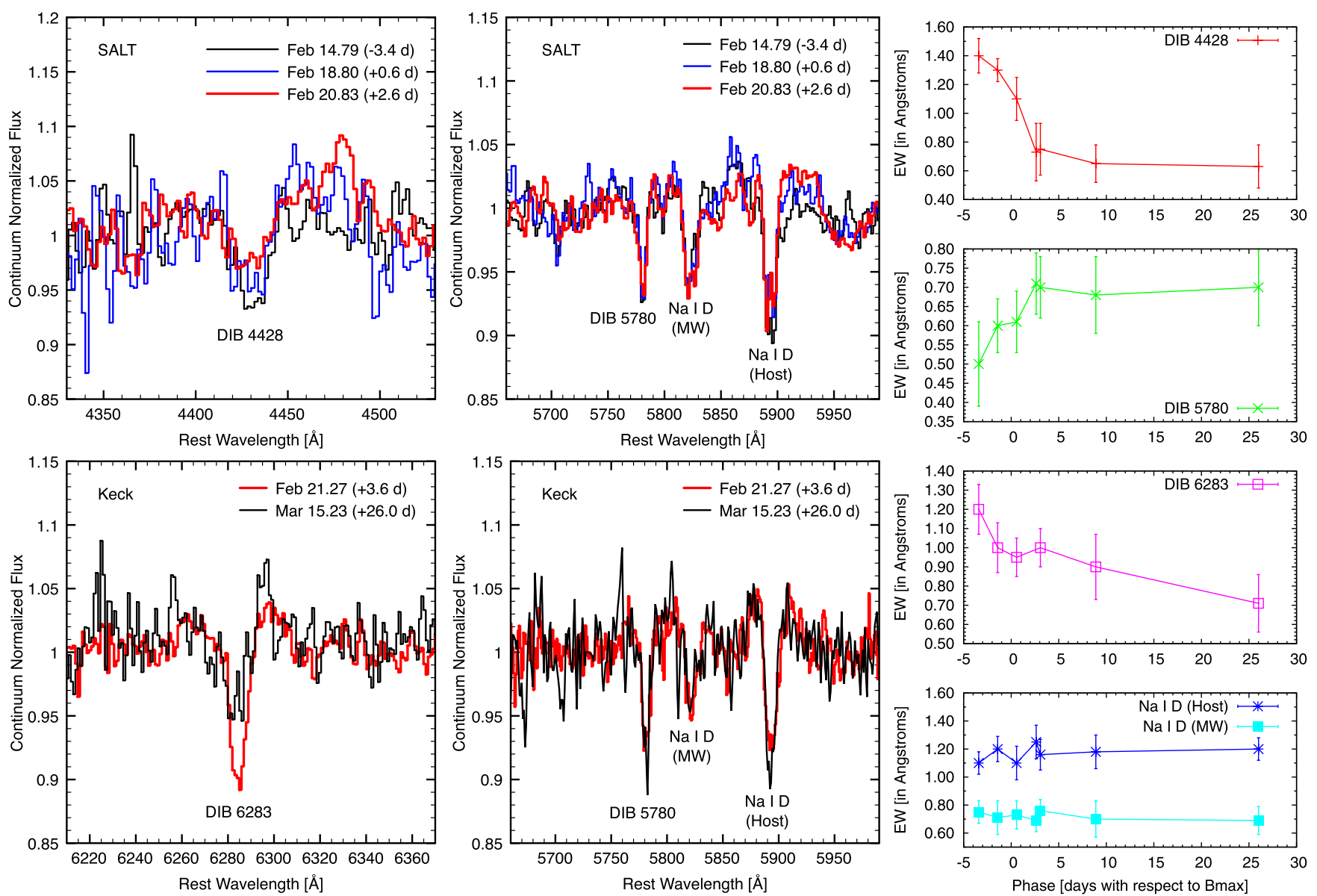

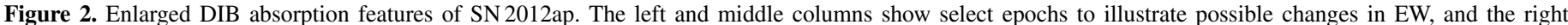

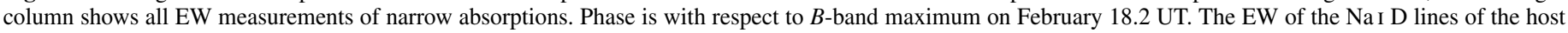

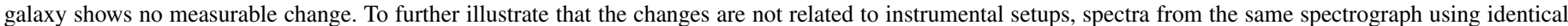
configurations are plotted.

(A color version of this figure is available in the online journal.)

The EW of DIB $\lambda 4428$ decreased by $0.77 \pm 0.25 \AA$ over $\sim 10$ days and DIB $\lambda 6283$ decreased by $0.49 \pm 0.28 \AA$ over $\sim 30$ days. The DIB $\lambda 5780$ feature, on the other hand, shows a weak but measurable increase of $\lesssim 0.2 \AA$ over $\sim 10$ days. The $\mathrm{Na}$ I $\mathrm{D}$ line at rest with respect to the host shows negligible change. The Na I D line associated with foreground Milky Way extinction shows no change, as expected.

\section{DISCUSSION}

\subsection{SN Interaction with DIB Carriers}

The DIB absorptions seen in the spectra of SN 2012ap are among the strongest extragalactic detections ever reported. Detections of extragalactic DIBs at this distance are rare and thus interesting as they allow one to compare Galactic ISM chemical properties with extragalactic ones. However, what is unique and most informative about the spectra of SN 2012ap is that the DIB absorption strengths change with time and that the changes are not uniform across different DIB features (see Figure 2).

Various types of interaction between the SN and DIB carrier material may explain the observed changes (see, e.g., Patat et al. 2010). We favor the scenario that the carrier material is nearby and the $\mathrm{SN}$ is actively interacting with it. This interaction can take many forms. Photons may modify or destroy carrier material via ionization and/or dissociation. If extremely nearby, the forward blast wave initiated by the explosion and traveling with velocity $\sim 0.4 c$ (S. Chakraborti et al., in preparation) will disrupt molecules and dust grains within a $\sim 0.01 \mathrm{pc}$ radius in the first 30 days.

\subsection{Physical Constraints on the DIB Carriers}

SN 2012ap peaked in the $B$ band on February 18.2 UT (D. Milisavljevic et al., in preparation), implying that the SN flux increased and then decreased at optical wavelengths as the intensities of DIB $\lambda 4428$ and $\lambda 6283$ became weaker and DIB $\lambda 5780$ became slightly stronger. This behavior is consistent with active interaction wherein separate DIB carriers differing in robustness and/or location are affected by the SN independently.

Using the time evolution of the blackbody temperature and total luminosity derived from photometry data (D. Milisavljevic et al., in preparation), we estimated the UV flux in the 5-50 eV spectral range as a function of distance and time from SN2012ap. To estimate the lifetimes of molecular material in this radiation field, we approximated the photoabsorption cross sections in this frequency range for small neutral molecules (Gallagher et al. 1988) and PAHs (Verstraete et al. 1990; Jochims et al. 1996), calculated the photoabsorption rate, and assumed that all absorption events lead to ionization or dissociation. Because these frequencies are above the peak of the 
blackbody curve, the absorption rates are highly sensitive to the ionization potential (IP) of a molecule, and the shape and size of its cross section.

The inferred lifetimes vary by several orders of magnitude, but within a distance of $\sim 0.01 \mathrm{pc}$, at peak luminosity all but the smallest neutral molecules are expected to have lifetimes much less than one day. Within this distance, the population of most neutral species will be rapidly depleted unless their formation from the breakdown of larger material is even more rapid. Cations, owing to their higher IPs, are estimated to have lifetimes on the order of days under the same conditions.

In this context, it is interesting to note that the timescale for the increase in DIB $\lambda 5780$ is comparable to that of the decay in DIB $\lambda 4428$, possibly suggesting that the DIB $\lambda 5780$ carrier is a photoproduct of the DIB $\lambda 4428$ carrier. In contrast, the decay in DIB $\lambda 6283$ occurs over a longer timescale, suggesting the carrier is either more photostable or is more extended. The ratio of the strength of DIB $\lambda 5780$ to DIB $\lambda 5797$ (the latter is not detected toward SN 2012ap) is positively correlated with increasing UV radiation environments (Vos et al. 2011). The increase in strength of DIB $\lambda 5780$ in these observations suggests that this trend continues to very extreme UV environments.

Fullerenes have been proposed as DIB carriers, and are significantly more stable against dissociation by UV radiation than smaller molecules, typically requiring energies of more than $10 \mathrm{eV}$ for dissociation (Diaz-Tendero et al. 2003). This increased dissociation energy might allow fullerenes to survive longer in the radiation environment around SN 2012ap. Using the photoabsorption cross section of $\mathrm{C}_{60}$ as a representative case (Berkowitz 1999), we estimate that neutral fullerenes (IP $\approx 7 \mathrm{eV}$ ) near SN2012ap will be rapidly ionized, but fullerene cations (IP $\sim 11 \mathrm{eV}$ ) should have lifetimes of order days. The fact that the observed changes in the EW of these DIB features occur on the timescale of days in such an intense UV field suggests that the carriers are fairly robust to ionization and dissociation (particularly DIB $\lambda 5780$ ), consistent with small cations or charged fullerenes.

\subsection{Implications of a DIB-SN Subtype Correlation}

Two other core-collapse $\mathrm{SNe}$ in the literature exhibit conspicuous DIBs in low-resolution spectra, and we examined their archival data: the Type Ib SN 2008D with spectra published by Modjaz et al. (2009), and the broad-lined Type Ib/c SN 2009bb published by Pignata et al. (2011). Figure 3 shows early-time spectra of these objects, with conspicuous DIB features highlighted. Although the relatively low spectral resolutions and limited temporal sampling prevent detailed analyses of these additional objects, the archival spectra suggest that some DIB features seen in these other SNe have both narrow and broad components and that they may vary as they do SN 2012ap.

All three SNe exhibited broad spectral features associated with ejecta moving at high velocities $\left(\gtrsim 2 \times 10^{4} \mathrm{~km} \mathrm{~s}^{-1}\right)$ within weeks of explosion and all were observed to have a color excess $E(B-V) \gtrsim 0.5$ mag that implies substantial extinction (Soderberg et al. 2008; Modjaz et al. 2009; Pignata et al. 2011; D. Milisavljevic et al., in preparation). SN 2012ap and SN 2009bb share similar explosion parameters of estimated ejecta mass $\left(\sim 2-4 M_{\odot}\right),{ }^{56} \mathrm{Ni}$ mass $\left(\sim 0.2 M_{\odot}\right)$, and explosion kinetic energy $\left(\sim 1.5 \times 10^{52} \mathrm{erg}\right)$. On the other hand, SN 2008D is different in that its broad lines disappeared within weeks as it transitioned to a SN Ib and its explosion energy $\left(\sim 1.5-6 \times 10^{51} \mathrm{erg}\right.$; Soderberg et al. 2008; Tanaka et al. 2009) is lower than those of SN 2012ap and SN 2009bb.

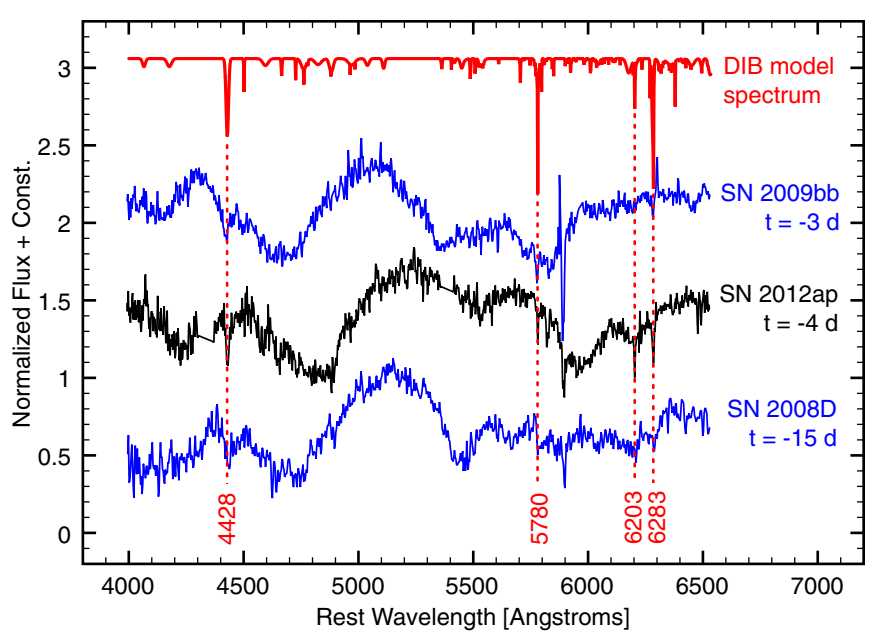

Figure 3. Model spectrum of known DIB absorption features compared to early-time spectra of SN 2012ap, SN 2009bb (Pignata et al. 2011), and SN 2008D (Modjaz et al. 2009). SN 2009bb and SN 2008D have been corrected for redshifts of $z=0.010$ and $z=0.007$, respectively. All SNe exhibit conspicuous absorptions having central wavelengths of well-known DIBs highlighted with vertical dashed lines. Time is with respect to maximum light. The model DIB spectrum was created from a catalog retrieved online at http://leonid.arc.nasa.gov/DIBcatalog.html.

(A color version of this figure is available in the online journal.)

Chance alignments between DIB-carrier-rich molecular clouds and these $\mathrm{SNe}$ are possible. However, given that the three SNe with conspicuous DIB absorptions examined in the literature are spectroscopically similar, it may be that the SN progenitor systems are related to the sources of the DIBs. If true, the carrier material responsible for the observed DIB absorptions in these $\mathrm{SNe}$ should lie fairly close to the explosion site and could be associated with mass loss from the progenitor star.

Mass loss in massive stars is influenced by a number of factors including the strength of their winds, rotation, the presence of a binary companion, possible eruptive mass-loss episodes, and environmental metallicity (Chiosi \& Maeder 1986; Humphreys \& Davidson 1994; Nugis \& Lamers 2000). To investigate what role metallicity might play in linking the three $\mathrm{SNe}$, the relative strengths of narrow lines from coincident host-galaxy emission at the site of SN2012ap were measured using the method described by Sanders et al. (2012). From the N2 diagnostic of Pettini \& Pagel (2004), we measure an oxygen abundance $\log (\mathrm{O} / \mathrm{H})+12=8.79$ with uncertainty 0.06 dex. Adopting a solar metallicity of $\log (\mathrm{O} / \mathrm{H})_{\odot}+12=8.69$ (Asplund et al. 2005), our measurement indicates that SN 2012ap exploded in an environment of around solar metallicity that lies in between the metallicity estimates of SN 2009bb (1.7-3.5 $Z_{\odot}$; Levesque et al. 2010) and SN 2008D (0.5-1 $Z_{\odot}$; Soderberg et al. 2008). Considering broad-lined SNe Ic are typically found in environments of subsolar metallicity (Kelly \& Kirshner 2012; Sanders et al. 2012), the metallicity of these three $\mathrm{SNe}$ is somewhat anomalous. However, these objects were discovered by surveys targeting high-mass metal-rich galaxies, so this weak trend may be influenced by an observational bias.

A handful of reports connect strong DIB features observed in a narrow subset of mass-losing stars with circumstellar shells (e.g., Tug \& Schmidt-Kaler 1981; Cohen \& Jones 1987). The circumstellar material is often nitrogen-rich and the strength of the associated DIB features may vary (Heydari-Malayeri et al. 1993). Le Bertre \& Lequeux (1993) identified Wolf-Rayet (WR) 
stars of the WN subtype and luminous blue variable (LBV) stars enriched in nitrogen as candidate objects with circumstellar shells containing DIB carriers, and proposed that nitrogen could act either as a constituent of the DIB carriers or as a catalyst for their production.

It is intriguing that families of WR and LBV stars may be associated with DIB features. WR stars are suspected progenitors of SNe Ib/c (Gaskell et al. 1986), and have been implicated for SN 2008D and SN 2009bb (Soderberg et al. 2008, 2010; Modjaz et al. 2009; Pignata et al. 2011). Although LBVs are not widely believed to be the direct progenitors of $\mathrm{SNe} \mathrm{Ib} /$ c, WR stars can evolve from a prior LBV phase (Conti 1976). These stars exhibit varying degrees of asymmetric mass loss (see, e.g., Nota et al. 1995), thus an observer's line of sight with respect to a circumstellar disk could be an important factor in explaining why strong DIB detections like those reported here are rare.

Finally, we note that varying strengths in narrow absorption lines attributable to interaction between an SN and a local environment has recently been recognized in a growing number of cases, with significant implications for the nature of the progenitor systems (e.g., Patat et al. 2007; Blondin et al. 2009; Dilday et al. 2012). However, those reports have been for Na I D, $\mathrm{Ca}$ II, $\mathrm{H} \alpha, \mathrm{He}$, and Fe II lines with line-of-sight blueshifted velocities of $\lesssim 100 \mathrm{~km} \mathrm{~s}^{-1}$ originating from circumstellar material around Type Ia SNe. This is not the same as what is being observed in the core-collapse SN 2012ap, where the DIB features are near zero velocity and are associated with a carrier material having radically different physical properties.

\section{CONCLUSIONS}

The broad-lined Type Ic SN 2012ap exhibits DIB absorptions that are among the strongest ever detected in an extragalactic object. The DIB features centered around $4428 \AA$, $5780 \AA$, and $6283 \AA$ undergo changes in EW over relatively short timescales $(t<30$ days) indicative of interaction between the SN and DIB carriers. Similar absorptions observed in archival spectra of two additional SNe suggest that SN 2012ap may belong to a subset of energetic $\mathrm{SNe} \mathrm{Ib} / \mathrm{c}$ that exhibit changes in conspicuous DIB absorption features. If true, this correlation is consistent with the DIB carrier-rich material being located close to the explosion, fairly resistant to the strong UV field, and potentially associated with mass loss of the progenitor star.

Our data with 4-7 $\AA$ resolution that monitored the spectral evolution of SN2012ap during its rise and fall in flux was on the cusp of detection for this uniquely strong source of DIB absorptions. Only the broadest DIB features known to have FWHM widths of approximately $2-12 \AA$ were observed in our data set. Thus, multi-epoch observations of $\mathrm{SNe}$ with spectral resolutions of $\leqslant 1 \AA$ beginning within days of explosion could uncover the presence of a larger family of DIB features. Such observations would be much more sensitive to possible variations in $\mathrm{Na}$ I absorption strength, as well as detect possible subtle changes in the velocities of the NaI/DIB features. Observed in this way, SNe with DIB absorptions have the potential to reveal unique information about the mass-loss environment of their progenitor systems and probe DIB carriers in new ways that can bring us closer to understanding their nature.

We thank an anonymous referee for a helpful, detailed, and critical reading of the Letter. T. Snow kindly provided comments on an early draft of the Letter. P. Massey provided insightful comments. G. Pignata, S. Valenti, D. Malesani, and G. Leloudas shared archival spectra that were examined. Many of the observations reported in this Letter were obtained with the Southern African Large Telescope. Additional data presented herein were obtained at the W. M. Keck Observatory, which is operated as a scientific partnership among the California Institute of Technology, the University of California, and NASA; the observatory was made possible by the generous financial support of the W. M. Keck Foundation. A. Miller, P. Nugent, and A. Morgan helped obtain the Keck observations. Some observations also came from the MMT Observatory, a joint facility of the Smithsonian Institution and the University of Arizona. Support was provided by the David and Lucile Packard Foundation Fellowship for Science and Engineering awarded to A.M.S. J.M.S. is supported by an NSF Astronomy and Astrophysics Postdoctoral Fellowship under award AST-1302771. T.E.P. thanks the National Research Foundation of South Africa. R.P.K. and J.C.W. are grateful for NSF grants AST-1211196 and AST-1109801, respectively. A.V.F. and S.B.C. acknowledge generous support from Gary and Cynthia Bengier, the Richard and Rhoda Goldman Fund, the Christopher R. Redlich Fund, the TABASGO Foundation, and NSF grant AST-1211916. K.N.C. has been supported by a CfA Postdoctoral Fellowship from the Smithsonian Astrophysical Observatory. This Letter made extensive use of the SUSPECT database (http://www.nhn.ou.edu/ suspect/).

\section{REFERENCES}

Asplund, M., Grevesse, N., \& Sauval, A. J. 2005, in ASP Conf. Ser. 336, Cosmic Abundances as Records of Stellar Evolution and Nucleosynthesis, ed. T. G. Barnes, III \& F. N. Bash (San Francisco, CA: ASP), 25

Berkowitz, J. 1999, JChPh, 111, 1446

Blondin, S., Prieto, J. L., Patat, F., et al. 2009, ApJ, 693, 207

Burgh, E. B., Nordsieck, K. H., Kobulnicky, H. A., et al. 2003, Proc. SPIE, 4841, 1463

Chiosi, C., \& Maeder, A. 1986, ARA\&A, 24, 329

Cohen, M., \& Jones, B. F. 1987, ApJL, 321, L151

Conti, P. S. 1976, MSRSL, 9, 193

Cordiner, M. A., Cox, N. L. J., Trundle, C., et al. 2008, A\&A, 480, L13

Cox, N. L. J. 2011, in Proc. of IAU Symp. Vol. 280, The Molecular Universe, ed. J. Cernicharo \& R. Bachiller (Cambridge: Cambridge Univ. Press), 162

Cox, N. L. J., Cordiner, M. A., Ehrenfreund, P., et al. 2007, A\&A, 470, 941

Cox, N. L. J., \& Patat, F. 2008, A\&A, 485, L9

Diaz-Tendero, S., Alcami, M., \& Martin, F. 2003, JChPh, 119, 5545

Dilday, B., Howell, D. A., Cenko, S. B., et al. 2012, Sci, 337, 942

Ellison, S. L., York, B. A., Murphy, M. T., et al. 2008, MNRAS, 383, L30

Gallagher, J. W., Brion, C. E., Samson, J. A. R., \& Langhoff, P. W. 1988, JPCRD, 17,9

Gaskell, C. M., Cappellaro, E., Dinerstein, H. L., et al. 1986, ApJL, 306, L77 Geballe, T. R., Najarro, F., Figer, D. F., Schlegelmilch, B. W., \& de La Fuente, D. 2011, Natur, 479, 200

Heger, M. L. 1922, LicOB, 10, 141

Herbig, G. H. 1995, ARA\&A, 33, 19

Heydari-Malayeri, M., Grebel, E. K., Melnick, J., \& Jorda, L. 1993, A\&A, 278, 11

Hobbs, L. M., York, D. G., Thorburn, J. A., et al. 2009, ApJ, 705, 32

Humphreys, R. M., \& Davidson, K. 1994, PASP, 106, 1025

Jewett, L., Cenko, S. B., Li, W., et al. 2012, CBET, 3037, 1

Jochims, H. W., Baumgartel, H., \& Lench, S. 1996, A\&A, 314, 1003

Kelly, P. L., \& Kirshner, R. P. 2012, ApJ, 759, 107

Le Bertre, T., \& Lequeux, J. 1993, A\&A, 274, 909

Levesque, E. M., Soderberg, A. M., Foley, R. J., et al. 2010, ApJL, 709, L26

Luna, R., Cox, N. L. J., Satorre, M. A., et al. 2008, A\&A, 480, 133

Merrill, P. W. 1934, PASP, 46, 206

Milisavljevic, D., Fesen, R., Soderberg, A., et al. 2012, CBET, 3037, 2

Modjaz, M., Li, W., Butler, N., et al. 2009, ApJ, 702, 226

Nota, A., Livio, M., Clampin, M., \& Schulte-Ladbeck, R. 1995, ApJ, 448, 788

Nugis, T., \& Lamers, H. J. G. L. M. 2000, A\&A, 360, 227

Oke, J. B., Cohen, J. G., Carr, M., et al. 1995, PASP, 107, 375

Patat, F., Chandra, P., Chevalier, R., et al. 2007, Sci, 317, 924

Patat, F., Cox, N. L. J., Parrent, J., \& Branch, D. 2010, A\&A, 514, A78 
Pettini, M., \& Pagel, B. E. J. 2004, MNRAS, 348, L59

Pignata, G., Stritzinger, M., Soderberg, A., et al. 2011, ApJ, 728, 14

Sanders, N. E., Soderberg, A. M., Levesque, E. M., et al. 2012, ApJ, 758,132

Sarre, P. J. 2006, JMoSp, 238, 1

Schmidt, G. D., Weymann, R. J., \& Foltz, C. B. 1989, PASP, 101, 713

Snow, T. P., Jr., \& Wallerstein, G. 1972, PASP, 84, 492

Soderberg, A. M., Berger, E., Page, K. L., et al. 2008, Natur, 453, 469

Soderberg, A. M., Chakraborti, S., Pignata, G., et al. 2010, Natur, 463, 513
Springob, C. M., Masters, K. L., Haynes, M. P., Giovanelli, R., \& Marinoni, C. 2009, ApJS, 182, 474

Tanaka, M., Tominaga, N., Nomoto, K., et al. 2009, ApJ, 692, 1131

Tug, H., \& Schmidt-Kaler, T. 1981, A\&A, 94, 16

Verstraete, L., Leger, A., Dhendecourt, L., Dutuit, O., \& Defourneau, D. 1990 A\&A, 237, 436

Vos, D. A. I., Cox, N. L. J., Kaper, L., Spaans, M., \& Ehrenfreund, P. 2011, A\&A, 533, A129

Xu, D., Zhang, J.-J., Chen, J., et al. 2012, ATel, 3922, 1 